\title{
Metabolomic profiling of uremic solutes in CKD patients
}

\author{
Takafumi Toyohara $^{1,7}$, Yasutoshi Akiyama ${ }^{1,7}$, Takehiro Suzuki ${ }^{1,7}$, Yoichi Takeuchi ${ }^{1}$, Eikan Mishima ${ }^{1}$, \\ Masayuki Tanemoto ${ }^{1}$, Ayako Momose ${ }^{2}$, Naoko Toki ${ }^{2}$, Hiroshi Sato ${ }^{1}$, Masaaki Nakayama ${ }^{3}$, Atsushi Hozawa ${ }^{4}$, \\ Ichiro Tsuji ${ }^{4}$, Sadayoshi Ito ${ }^{1}$, Tomoyoshi Soga ${ }^{2}$ and Takaaki Abe ${ }^{1,5,6}$
}

Early detection and accurate monitoring of patients with chronic kidney disease (CKD) is likely to improve care and decrease the risk of cardiovascular and cerebrovascular diseases. As a new diagnostic tool, we examined the retention of uremic solutes as a simpler, more accurate method to assess renal function. To achieve this, we comprehensively evaluated these solutes in CKD patients. By capillary electrophoresis with mass spectrometry, we found 22 cations and 30 anions that accumulated significantly as the estimated glomerular filtration rate (eGFR) decreased. These compounds included 9 cations and 27 anions that were newly identified in this study. In contrast, we also found 7 cations ( 2 new) and 5 anions (all new) that decrease significantly as eGFR declines. We evaluated each substance for its suitability to detect early CKD stage. Compounds that are highly correlated with eGFR and whose plasma concentration changed in a manner approximated by the first-degree equation are excellent candidates for detecting CKD and identifying uremic toxins that might aggravate kidney function in the early stage of CKD. These results identify a number of uremic compounds, many of which are novel and which predict worsening renal function. These compounds provide diagnostic information and may be targets for therapies designed to treat the complications of CKD patients.

Hypertension Research (2010) 33, 944-952; doi:10.1038/hr.2010.113; published online 8 July 2010

Keywords: biomarker; CE-MS; CKD; uremic toxin

\section{INTRODUCTION}

Chronic kidney disease (CKD) is an important public health problem and determination of the glomerular filtration rate (GFR) has been essential for the evaluation of CKD patients. ${ }^{1-3}$ Early detection of impairment of renal function allows treatment to prevent further deterioration and complications. With the progression of CKD, various uremic toxins accumulate, subsequently causing renal damage and hypertension. ${ }^{4,5}$ Recently, we revealed that many compounds accumulate during renal failure, and that the kidney-specific organic anion transporter SLCO4C1 excretes uremic toxins, resulting in the reduction of blood pressure and renal inflammation. ${ }^{6}$ To generalize these results for clinical use, it is necessary to examine the accumulation of uremic solutes precisely. Therefore, the aims of this study were (1) to identify new uremic retention solutes that accumulate in CKD patients comprehensively by capillary electrophoresis with mass spectrometry (CE-MS) and (2) to determine substances that are more sensitive for detecting early renal damage than serum creatinine $(\mathrm{Cr})$ or cystatin $\mathrm{C}$.

\section{MATERIALS AND METHODS}

Study population

The study population comprised 41 CKD patients who visited the outpatient clinic of Tohoku University Hospital between April 2007 and December 2008.
The study protocol complied with the Declaration of Helsinki and was approved by the Committees on the Ethics of Human Research of Tohoku University. Informed consent was obtained from each participant. Cystatin C was measured using a BNII nephelometer (Dade Behring, currently Siemens Healthcare Diagnostics, Deerfield, IL, USA) and a particle-enhanced immunonephelometric assay (N Latex cystatin C; Siemens Healthcare Diagnostics). Biochemical analyses were carried out using routine methods at the Department of Clinical Chemistry at Tohoku University Hospital.

\section{CE-MS measurement for metabolome analysis}

A comprehensive and quantitative analysis of charged metabolites by CE-MS was performed. ${ }^{6-10} \mathrm{CKD}$ patient's plasma $(50 \mu \mathrm{l})$ was immediately plunged into methanol $(450 \mu \mathrm{l})$ containing internal standards $(20 \mu \mathrm{M}$ each of methionine sulfone; Wako, Osaka, Japan) for cations, MES (Dojindo, Kumamoto, Japan) and CSA (D-camphol-10-sulfonic acid; Wako). Deionized water $(200 \mu \mathrm{l})$ and chloroform $(500 \mu \mathrm{l})$ were then added, and the mixture was thoroughly mixed. The solution was centrifuged at $4600 \times g$ for $5 \mathrm{~min}$ at $4{ }^{\circ} \mathrm{C}$, and the upper aqueous layer was centrifugally filtered through a Millipore $5000 \mathrm{Da}$ cutoff filter (Millipore, Billerica, MA, USA) to remove proteins. The filtrate was lyophilized and dissolved in $25 \mu \mathrm{l}$ of Milli-Q water containing reference compounds ( $200 \mu_{\mathrm{M}}$ each of 3-aminopyrrolidine; Sigma-Aldrich, St Louis, MO, USA) and trimesate (Wako) before CE-TOFMS (capillary electrophoresis with electrospray ionization time-of-flight mass spectrometry) analysis. All CE-TOFMS experiments were performed using the Agilent CE capillary

${ }^{1}$ Division of Nephrology, Endocrinology, and Vascular Medicine, Tohoku University Graduate School of Medicine, Sendai, Japan; ${ }^{2}$ Institute for Advanced Biosciences, Keio University, Tsuruoka, Japan; ${ }^{3}$ Research Division of Dialysis and Chronic Kidney Disease, Tohoku University Graduate School of Medicine, Sendai, Japan; ${ }^{4}$ Department of Public Health, Tohoku University Graduate School of Medicine, Sendai, Japan; ${ }^{5}$ Division of Medical Science, Tohoku University Graduate School of Biomedical Engineering, Sendai, Japan and ${ }^{6}$ Department of Clinical Biology and Hormonal Regulation, Tohoku University Graduate School of Medicine, Sendai, Japan

7These authors contributed equally to this work.

Correspondence: Dr T Abe, Division of Medical Science, Tohoku University Graduate School of Biomedical Engineering, Department of Clinical Biology and Hormonal Regulation, Tohoku University Graduate School of Medicine, Sendai 980-8574, Japan.

E-mail: takaabe@med.tohoku.ac.jp

Received 10 February 2010; revised 1 April 2010; accepted 27 April 2010; published online 8 July 2010 
Table 1 Baseline characteristics of 41 CKD patients

\section{Age}

$\operatorname{Sex}(M / F)$

Cystatin C (mg per $100 \mathrm{ml}$ )

Creatinine (mg per $100 \mathrm{ml}$ )

eGFR ( $\mathrm{ml} \mathrm{min}^{-1}$ per $1.73 \mathrm{~m}^{2}$ )

Urine protein $\left(\mathrm{ggCr}^{-1}\right)$

Uric acid (mg per $100 \mathrm{ml}$ )

Hemoglobin (g per $100 \mathrm{ml}$ )

Height (cm)

Weight (kg)

Systolic blood pressure $(\mathrm{mm} \mathrm{Hg})$

Diastolic blood pressure $(\mathrm{mm} \mathrm{Hg})$

\section{Cause of CKD}

Alport syndrome

Analgesic-induced nephropathy

ANCA-related nephropathy

Aortitis syndrome

Bilateral renal infarction

Diabetic nephropathy

Focal segmental glomerulosclerosis

Glomeruloscrelosis

Hydronephrosis

IgA nephropathy

Lipoprotein glomerulopathy

Lupus nephropathy

Membrane proliferative glomerulonephritis

Membranous nephropathy

Polycystic kidney

Renal tubercurosis

Sarcoidosis

Unknown

\section{Baseline disease}

Cardiovascular disease

Diabetes mellitus

Hypertension

Smoking

Body mass index ( $>30 \mathrm{~kg} \mathrm{~m}^{-2}$ )

Drug

Allopurinol

Angiotensin receptor blocker

AST-120

Erythropoietin

Statin
Hyperlipidemia

$62.8+14.6(27-84)$

$16 / 25$

$2.29 \pm 0.90(0.96-4.11)$

$2.64 \pm 1.60(0.8-7.4)$

$24.4 \pm 13.9(5.9-57.0)$

$1.58 \pm 1.64(0.00-7.07)$

$7.68 \pm 1.57$ (4.4-9.8)

$11.28 \pm 2.06(7.1-17.5)$

$158.0 \pm 7.57(140.1-175)$

$57.2 \pm 10.0(42-72.5)$

$129.07 \pm 14.21$ (107-160)

$76.29 \pm 11.66(53-110)$

$1(2.4 \%)$

$1(2.4 \%)$

$1(2.4 \%)$

$1(2.4 \%)$

$1(2.4 \%)$

$8(19.5 \%)$

$3(7.3 \%)$

$4(9.7 \%)$

$1(2.4 \%)$

$9(21.9 \%)$

$1(2.4 \%)$

$2(4.8 \%)$

$1(2.4 \%)$

$2(4.8 \%)$

$1(2.4 \%)$

$1(2.4 \%)$

$1(2.4 \%)$

$2(4.8 \%)$

Abbreviations: ANCA, antineutrophil cytoplasmic autoantibody; CKD, chronic kidney disease eGFR, estimated glomerular filtration rate.

electrophoresis system (Agilent Technologies, Waldbronn, Germany), the Agilent G3250AA LC/MSD TOF system (Agilent Technologies, Palo Alto, CA, USA), the Agilent 1100 series binary high performance liquid chromatography pump, the G1603A Agilent CE-MS adapter and the G1607A Agilent CE-ESIMS sprayer kit. For data acquisition, we used G2201AA Agilent ChemStation software for CE and Analyst QS for Agilent TOFMS software. ${ }^{9}$

\section{Data analysis}

GFR was estimated using the simplified prediction equation derived from the modified version described in the Modification of Diet in Renal Disease Study as proposed by the Japanese Society of Nephrology: ${ }^{11} \mathrm{eGFR}=194 \times[\text { age }]^{-0.287} \times[$ serum creatinine $(\mathrm{mg}$ per $100 \mathrm{ml})]^{-1.094} \times[0.739$ if female $]$. All values are expressed as
Table 2 The $\mathbf{4 2}$ cationic compounds detected by CE-MS that negatively correlated with eGFR

\begin{tabular}{|c|c|c|c|}
\hline Compound & $\begin{array}{l}\text { Spearman's correlation } \\
\text { coefficients }\end{array}$ & P-value & Reference \\
\hline Creatinine & -0.965 & $<0.001$ & 5,6 \\
\hline SDMA & -0.946 & $<0.001$ & 5,14 \\
\hline Guanidinosuccinate & -0.933 & $<0.001$ & $5,6,15$ \\
\hline Citrulline & -0.801 & $<0.001$ & $6,16,17$ \\
\hline 1-Methyladenosine & -0.772 & $<0.001$ & 5,18 \\
\hline N-Acetylglucosamine & -0.751 & $<0.001$ & \\
\hline$\gamma$-Butyrobetaine & -0.734 & $<0.001$ & \\
\hline Ophthalmate & -0.687 & $<0.001$ & \\
\hline 3-Methylhistidin & -0.669 & $<0.001$ & $6,16,17$ \\
\hline Hydroxyproline & -0.666 & $<0.001$ & 19 \\
\hline Trimethylamine $\mathrm{N}$-oxide & -0.665 & $<0.001$ & 6,20 \\
\hline Allantoin & -0.653 & $<0.001$ & 6,21 \\
\hline ADMA & -0.614 & $<0.001$ & $5,6,14$ \\
\hline$N$ - $\varepsilon$-Acetyllysine & -0.602 & $<0.001$ & \\
\hline Kynurenine & -0.579 & $<0.001$ & 5,22 \\
\hline Cytosine & -0.570 & $<0.001$ & \\
\hline Indole-3-acetate & -0.475 & 0.002 & 5,23 \\
\hline Hypotaurine & -0.447 & 0.004 & \\
\hline$N, N$-Dimethylglycine & -0.428 & 0.006 & 6,24 \\
\hline 7-Methylguanine & -0.406 & 0.010 & \\
\hline Methionine sulfoxide & -0.405 & 0.010 & $6^{a}$ \\
\hline Asn & -0.337 & 0.033 & \\
\hline Cysteine-glutathione disulphide & -0.279 & 0.077 & \\
\hline Gln & -0.273 & 0.083 & \\
\hline Arg & -0.271 & 0.087 & \\
\hline $\mathrm{His}$ & -0.267 & 0.091 & \\
\hline Ethanolamine phosphate & -0.261 & 0.098 & \\
\hline Gly & -0.258 & 0.102 & \\
\hline Piperazine & -0.253 & 0.110 & \\
\hline$\beta-A l a$ & -0.243 & 0.124 & \\
\hline Ornithine & -0.192 & 0.244 & 17 \\
\hline Homoarginine; & -0.183 & 0.248 & \\
\hline \multicolumn{4}{|l|}{ N6-N6-N6-trimethyllysine } \\
\hline Sarcosine & -0.182 & 0.250 & \\
\hline Pipecolate & -0.122 & 0.439 & $6^{b}$ \\
\hline O-Acetylcarnitine & -0.118 & 0.453 & \\
\hline Pro & -0.113 & 0.474 & \\
\hline Butanoate & -0.108 & 0.491 & \\
\hline 5-Methyltetrahydrofolate & -0.088 & 0.576 & \\
\hline Ala & -0.070 & 0.656 & \\
\hline 5-Oxoproline & -0.032 & 0.837 & \\
\hline Taurine & -0.024 & 0.879 & 17 \\
\hline Ser & -0.0003 & 0.998 & \\
\hline
\end{tabular}

Abbreviations: ADMA, asymmetric dimethylarginine; CE-MS, capillary electrophoresis with mass spectrometry; CKD, chronic kidney disease; eGFR, estimated glomerular filtration rate; SDMA, symmetric dimethylarginine.

These compounds are lined in an ascending order of Spearman's correlation coefficients.

A value of $P<0.05$ is accepted as indicating significant correlation with eGFR (22 cations). Shaded compounds have not been reported before. Methionine sulfoxide was increased in CKD patients, although it decreased in rat renal failure. Pipecolate was increased in rat renal failure; however, it was not increased significantly in CKD patients.

a Decreased.

bIncreased.

mean \pm s.d. The statistical analysis was performed using statistical software SPSS II version 11 (Chicago, IL, USA). Spearman's rank correlation was calculated to assess the correlation between eGFR and the plasma concentrations of the compounds. A value of $P<0.05$ was accepted as indicating statistical significance.

To estimate the log likelihood, we used PROC GENMOD in SAS software version 9 (SAS Institute, Cary, NC, USA). We assumed that the compounds might fit either the first-degree or second-degree model. The first-degree model 
Table 3 The 24 cationic compounds detected by CE-MS that are positively correlated with eGFR (that is, the plasma concentrations of these compounds are decreased as eGFR declines)

\begin{tabular}{|c|c|c|c|}
\hline Compound & $\begin{array}{c}\text { Spearman's correlation } \\
\text { coefficients }\end{array}$ & P-value & Reference \\
\hline $\operatorname{Trp}$ & 0.668 & $<0.001$ & 6,16 \\
\hline Val & 0.478 & 0.002 & 17 \\
\hline Tyr & 0.453 & 0.004 & $6,16,17$ \\
\hline 2-Aminobutyrate & 0.453 & 0.004 & \\
\hline Guanidoacetate & 0.441 & 0.005 & 6,15 \\
\hline Glu & 0.422 & 0.007 & \\
\hline Leu & 0.386 & 0.014 & 17 \\
\hline Asp & 0.262 & 0.097 & \\
\hline Hypoxanthine & 0.259 & 0.101 & \\
\hline Ile & 0.225 & 0.153 & \\
\hline Lys & 0.219 & 0.166 & \\
\hline Glycerophosphorylcholine & 0.211 & 0.182 & \\
\hline Guanosine & 0.207 & 0.190 & \\
\hline 1-Methyl-2-pyrrolidone & 0.207 & 0.190 & \\
\hline Carnitine & 0.202 & 0.201 & $6^{\mathrm{a}}$ \\
\hline Inosine & 0.193 & 0.223 & \\
\hline Urocanate & 0.181 & 0.251 & \\
\hline 1-Methylnicotinamide & 0.165 & 0.294 & \\
\hline Thr & 0.152 & 0.336 & \\
\hline Met & 0.121 & 0.444 & \\
\hline Betaine & 0.098 & 0.535 & \\
\hline Creatine & 0.084 & 0.593 & \\
\hline Phe & 0.058 & 0.712 & \\
\hline$\alpha$-Aminoadipate & 0.045 & 0.775 & \\
\hline
\end{tabular}

Abbreviations: CE-MS, capillary electrophoresis with mass spectrometry; CKD, chronic kidney disease; eGFR, estimated glomerular filtration rate.

These compounds are lined in descending order of Spearman's correlation coefficients. A value of $P<0.05$ is accepted as indicating significant correlation with eGFR (seven cations). Shaded compounds have not been reported before. Carnitine was decreased in rat renal failure; however, it was not increased significantly in CKD patients. a Decreased.

Table 4 The 38 anionic compounds detected by CE-MS that are negatively correlated with eGFR (that is, the plasma concentrations of these compounds are increased as eGFR declines)

\begin{tabular}{|c|c|c|c|}
\hline Compound & $\begin{array}{c}\text { Spearman's correlation } \\
\text { coefficients }\end{array}$ & P-value & Reference \\
\hline Isethionate & -0.929 & $<0.001$ & \\
\hline Gluconate & -0.929 & $<0.001$ & \\
\hline trans-Aconitate & -0.869 & $<0.001$ & 6 \\
\hline Pimelate & -0.847 & $<0.001$ & \\
\hline 3-Indoxyl sulfate & -0.834 & $<0.001$ & $5,6,26$ \\
\hline isocitrate & -0.825 & $<0.001$ & \\
\hline$N$-Acetyl- $\beta$-alanine & -0.805 & $<0.001$ & \\
\hline$N$-Acetylglutamate & -0.782 & $<0.001$ & 27 \\
\hline Sebacate & -0.751 & $<0.001$ & \\
\hline 4-Oxopentanoate & -0.725 & $<0.001$ & \\
\hline cis-Aconitate & -0.719 & $<0.001$ & \\
\hline Homovanillate & -0.711 & $<0.001$ & \\
\hline Adipate & -0.677 & $<0.001$ & \\
\hline Citramalate & -0.670 & $<0.001$ & \\
\hline 2-Isopropylmalate & -0.669 & $<0.001$ & \\
\hline Threonate & -0.631 & $<0.001$ & \\
\hline Hippurate & -0.608 & $<0.001$ & 5,28 \\
\hline $\mathrm{N}$-Acetylaspartate & -0.594 & $<0.001$ & \\
\hline 4-Hydroxy-3-methoxymandelate & -0.572 & $<0.001$ & \\
\hline
\end{tabular}

Table 4 Continued

\begin{tabular}{|c|c|c|c|}
\hline Compound & $\begin{array}{c}\text { Spearman's correlation } \\
\text { coefficients }\end{array}$ & P-value & Reference \\
\hline Oxamate & -0.559 & $<0.001$ & \\
\hline Glutarate & -0.465 & 0.003 & \\
\hline Azelate & -0.463 & 0.003 & \\
\hline Phthalate & -0.456 & 0.003 & \\
\hline Citrate & -0.454 & 0.004 & 29 \\
\hline Malonate & -0.440 & 0.005 & \\
\hline Citraconate & 0.401 & 0.011 & \\
\hline Quinate & -0.392 & 0.013 & \\
\hline Succinate & -0.362 & 0.021 & \\
\hline Cysteine S-sulfate & -0.343 & 0.030 & \\
\hline 4-Hydroxy-3-methoxybenzoate & -0.322 & 0.041 & \\
\hline Glycerophosphate & -0.284 & 0.072 & \\
\hline 2-Hydroxyoctarate & -0.208 & 0.189 & \\
\hline Malate & -0.157 & 0.321 & 29 \\
\hline 2-Hydroxypentanoate & -0.139 & 0.379 & $6^{a}$ \\
\hline Heptanoate & -0.128 & 0.416 & \\
\hline Laurate & -0.100 & 0.527 & \\
\hline 5-Oxoproline & -0.067 & 0.671 & \\
\hline Mucate & -0.064 & 0.686 & \\
\hline
\end{tabular}

Abbreviations: CE-MS, capillary electrophoresis with mass spectrometry; CKD, chronic kidney disease; eGFR, estimated glomerular filtration rate.

These compounds are lined in ascending order of Spearman's correlation coefficients. A value of $P<0.05$ is a copted as indicating significant correlation with eGFR ( 30 anions). Shaded compounds have not been reported before. Although 2-hydroxypentanoate was increased in rat renal failure, it was not increased significantly in CKD patients. ancreased.

Table 5 The 13 anionic compounds detected by CE-MS that are positively correlated with eGFR (that is, the plasma concentrations of these compounds are decreased as eGFR declines)

\begin{tabular}{lcr}
\hline Compound & $\begin{array}{c}\text { Spearman's correlation } \\
\text { coefficients }\end{array}$ & P-value \\
\hline 4-Methyl-2-oxopentanoate & 0.673 & $<0.001$ \\
2-Oxoisopentanoate & 0.645 & $<0.001$ \\
Lactate & 0.349 & 0.027 \\
Octanoate & 0.348 & 0.027 \\
2-Oxoglutarate & 0.331 & 0.036 \\
Pyruvate & 0.305 & 0.053 \\
2-Hydroxyisobutyrate, & 0.226 & 0.152 \\
2-hydroxybutyrate & & \\
Fumarate & 0.155 & 0.327 \\
3-Hydroxybutyrate & 0.094 & 0.553 \\
Pelargonate & 0.063 & 0.689 \\
Pentanoate, 3-methylbutanoate & 0.021 & 0.892 \\
Decanoate & 0.015 & 0.923 \\
Hexanoate & 0.011 & 0.946
\end{tabular}

Abbreviations: CE-MS, capillary electrophoresis with mass spectrometry; eGFR, estimated glomerular filtration rate.

These compounds are lined in ascending order of Spearman's correlation coefficients. A value of $P<0.05$ is accepted as indicating significant correlation with eGFR (five anions). Shaded compounds have not been reported before.

alncreased.

fits the compound concentrations in plasma of CKD patients $(\mathrm{Cp})=\beta 1(\mathrm{eGFR})+\alpha$, and the second-degree model fits $\mathrm{Cp}=\beta 1(\mathrm{eGFR})^{2}+\beta 2(\mathrm{eGFR})+\alpha$. The $\beta 1$ and $\beta 2$ are calculated using regression models. To estimate the suitability of both the first-degree and second-degree models, the appropriateness of the addition of eGFR $^{2}$ as an independent variable was examined by comparing the log likelihood ratios of the models. ${ }^{12,13}$ The difference between the log likelihood ratios is used for testing the significance of the contribution of $\mathrm{eGFR}^{2}$ to 
the model. ${ }^{13}$ This value follows $X^{2}$ distribution with 1 d.f. If the calculated difference is $>3.84$, we consider that the compound is more likely to fit the second-degree equation. To compare the plasma concentration change between the first-degree and second-degree models in the same graph, we converted $\mathrm{Cp}$ to Cpsd by calculating Z-scores: $\mathrm{Cpsd}=(\mathrm{Cp}-$ mean of $\mathrm{Cp}) / \mathrm{s}$.d. of $\mathrm{Cp}$. Cpsd means how many folds of s.d. the plasma concentration separates from the mean value.

\section{RESULTS}

Study population and CE-MS analysis

The characteristics of the patients are in Table 1. Using CE-MS, 312 cationic and 193 anionic compounds, whose molecular weights from 73.0 to $460.1 \mathrm{~m} / \mathrm{z}$ were detectable, and 65 cationic and 52
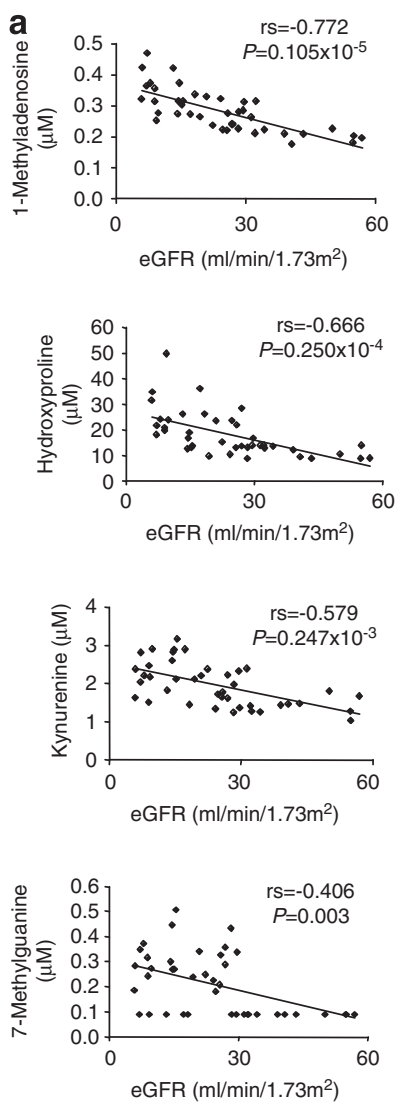

b
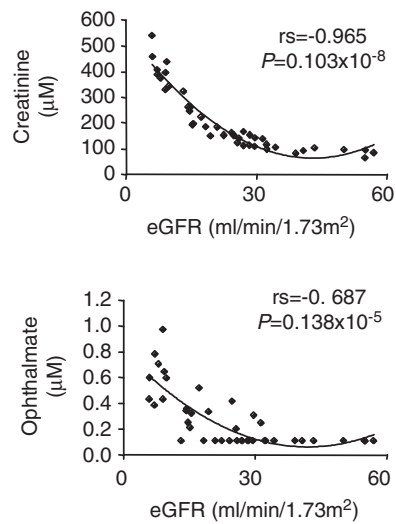
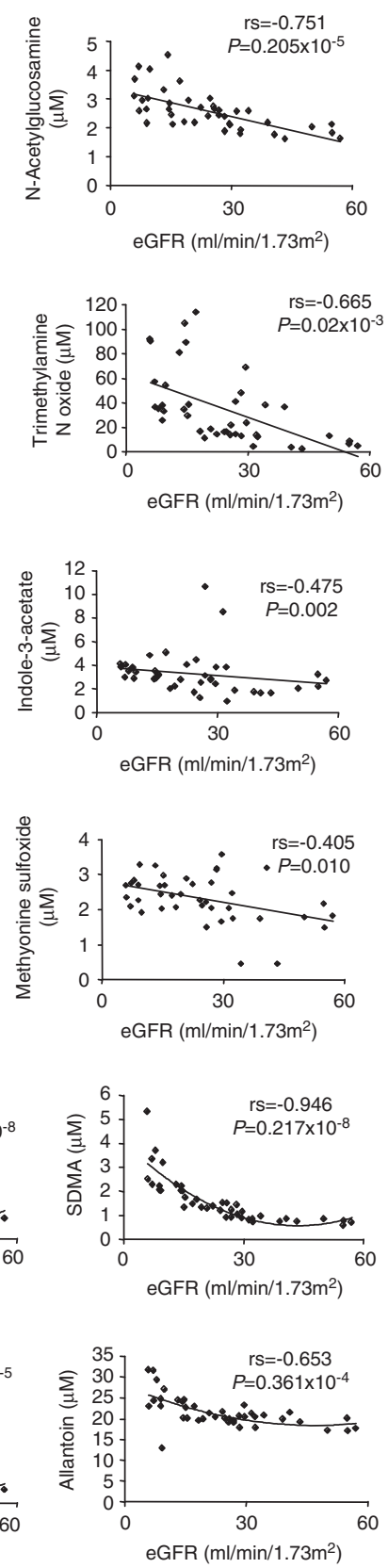

anionic compounds were identified in CKD patients (Tables 2-5). To find candidates that might be useful in detecting CKD, we analyzed the relationship between the compound concentrations and eGFR. As in Table 2, 42 cationic compounds were negatively correlated with eGFR (that is, the plasma concentrations of these compounds increased as eGFR declined), and the 22 cationic compounds were significant $(P<0.05)$. Among 22 compounds, 13 compounds were already reported as uremic retention solutes. ${ }^{5,14-24}$ On the other hand, other nine cationic compounds, $N$-acetylglucosamine, $\gamma$-butyrobetaine, ophthalmate, $N$ - $\varepsilon$-acetyllysine, cytosine, hypotaurine, 7-methylguanine, methionine sulfoxide and Asn, have not been reported to be accumulated (shaded in Table 2).
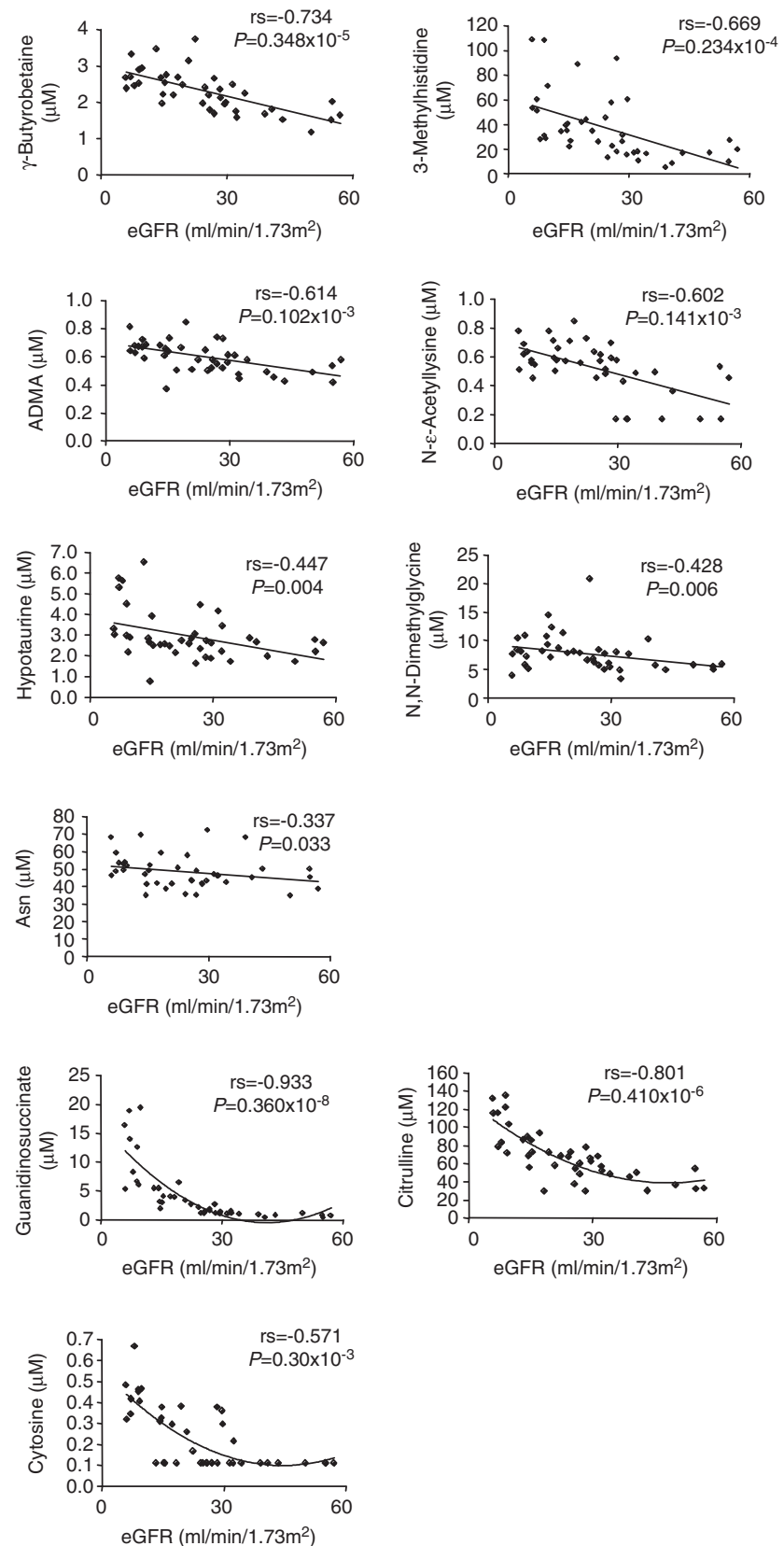

Figure 1 (a, b) The relation between eGFR and plasma concentration of 22 cationic compounds that accumulated significantly as eGFR declined is shown. (a) Compounds that are categorized in the first-degree equation group. (b) Compounds that are categorized in the second-degree equation group. (c, d) The relation between eGFR and plasma concentration of seven cationic compounds that accumulated significantly as eGFR declined is shown. (c) Compounds that are categorized in the first-degree equation group. (d) Compounds that are categorized in the second-degree equation group. 

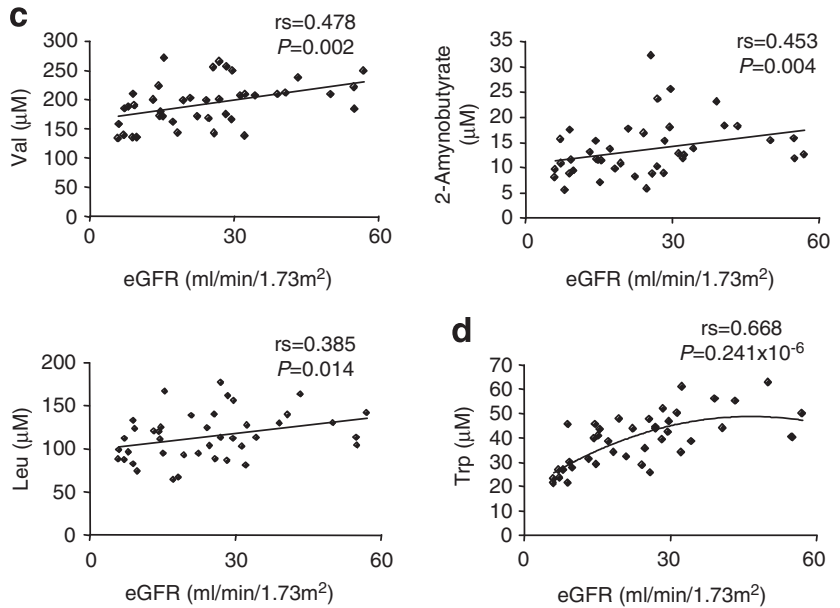

Figure 1 Continued.
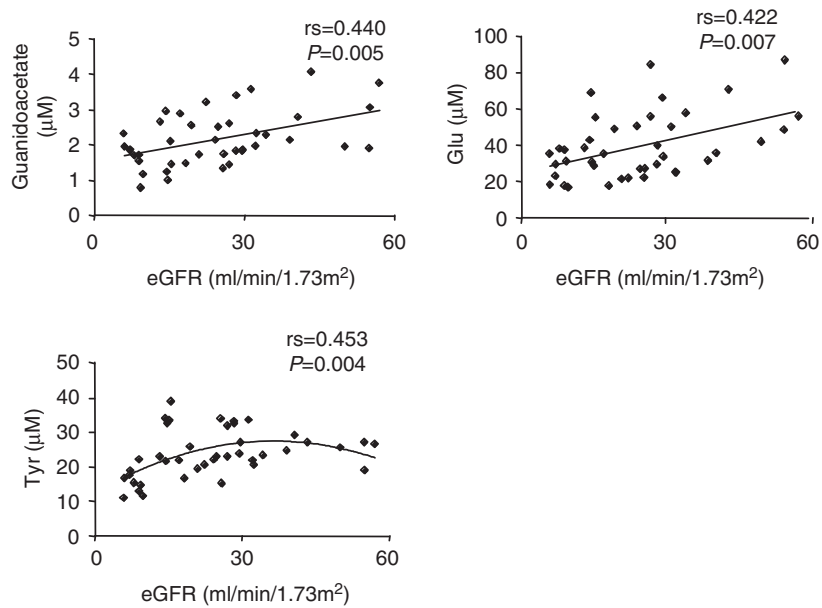

In Table 3, 24 cationic compounds were positively correlated with eGFR (that is, the plasma concentrations of these compounds were decreased as eGFR declined). Among these, seven cationic compounds, Trp, Val, Tyr, 2-aminobutyrate, guanidoacetate, Glu and Leu, were significantly correlated with eGFR. Five of these were previously reported, ${ }^{16,17,25}$ but 2 -aminobutyrate and Glu have not been known as compounds that decreased (shaded in Table 3 ).

In Table 4, 38 anionic compounds that negatively correlated with eGFR are shown. Among these, 30 anionic compounds were significantly correlated with eGFR.

Five anionic compounds, 3 -indoxyl sulfate, $\mathrm{N}$-acetylglutamate, trans-aconitate, hippurate and citrate, were already reported to increase in renal failure. ${ }^{5,6,26-29}$ On the other hand, 25 other compounds, isethionate, gluconate, pimelate, isocitrate, $N$-acetyl- $\beta$-alanine, sebacate, 4-oxopentanoate, cis-aconitate, homovanillate, adipate, citramalate, 2-isopropylmalate, threonate, $\mathrm{N}$-acetylaspartate, 4-hydroxy-3methoxymandelate, oxamate, glutarate, azelate, phthalate, malonate, citraconate, quinate, succinate, cysteine $S$-sulfate and 4-hydroxy-3methoxybenzoate, were clarified as new uremic retention anions (shaded in Table 4). In Table 5, 13 cationic compounds that positively correlated with eGFR are shown. Among these, five compounds, 4-methyl-2-oxopentanoate, 2-oxoisopentanoate, lactate, octanoate and 2-oxoglutarate, were significantly correlated with eGFR and the plasma concentrations of these five anions have not been reported to be decreased as eGFR declines (shaded in Table 5). These data identified various uremic solutes that correlated with the change of eGFR in CKD patients.

\section{Comparison of manner of change during renal damage}

We next examined whether the identified uremic retention solutes would be better markers for detecting early stage of CKD than $\mathrm{Cr}$ or cystatin $\mathrm{C}$ by fitting the first-degree or the second-degree equation models. ${ }^{13}$ Figures 1a and $\mathrm{b}$ show the relationship between eGFR and the plasma concentration of 22 cationic compounds accumulating significantly as eGFR declines. Among these, 15 compounds, 1-methyladenosine, $\mathrm{N}$-acetylglucosamine, $\gamma$-butyrobetaine, 3-methylhistidine, hydroxyproline, trimethylamine $N$-oxide, asymmetric dimethylarginine, $N$ - $\varepsilon$-acetyllysine, kynurenine, indole-3-acetate, hypotaurine, $\mathrm{N}, \mathrm{N}$-dimethylglycine, 7-methylguanine, methionine sulfoxide and Asn, were approximated and categorized in the first-degree equation group (Figure 1a). This means that the concentrations of these compounds increased linearly from eGFR 60 to $10 \mathrm{ml} \mathrm{min}^{-1}$ per
$1.73 \mathrm{~m}^{2}$. On the other hand, seven compounds, $\mathrm{Cr}$, symmetric dimethylarginine, guanidinosuccinate, citrulline, ophthalmate, allantoin and cytosine, were categorized in the second-degree equation group (Figure $1 \mathrm{~b}$ ). Figures $1 \mathrm{c}$ and $\mathrm{d}$ show the positive relation between eGFR and the plasma concentration of seven cationic compounds. Val, 2-aminobutyrate, guanidoacetate, Glu and Leu were categorized and approximated in the first-degree equation group (Figure 1c). This means that the concentration of these compounds decreased linearly from eGFR 60 to $10 \mathrm{ml} \mathrm{min}^{-1}$ per $1.73 \mathrm{~m}^{2}$. On the other hand, Trp and Try were categorized in the second-degree equation group (Figure 1d).

Figures $2 \mathrm{a}$ and $\mathrm{b}$ show the relation between eGFR and the plasma concentration of 30 anionic compounds, which accumulated significantly as eGFR declined. Sebacate, cis-aconitate, homovanillate, adipate, citramalate, 2-isopropylmalate, $\mathrm{N}$-acetylaspartate, 4-hydroxy-3methoxymandelate, oxamate, glutarate, azelate, phthalate, citrate, malonate, citraconate, quinate, succinate, cysteine $S$-sulfate and 4-hydroxy-3-methoxybenzoate were categorized in the group approximated by the first-degree equation and negatively correlated with eGFR (Figure 2a). On the other hand, isethionate, gluconate, transaconitate, pimelate, 3 -indoxyl sulfate, isocitrate, $N$-acetyl- $\beta$-alanine, $\mathrm{N}$-acetylglutamate, 4-oxopentanoate, threonate and hippurate were categorized in the second-degree equation group (Figure $2 b$ ).

Figures $2 \mathrm{c}$ and $\mathrm{d}$ show the relation between eGFR and the plasma concentration of five anionic compounds that decreased significantly as eGFR declined. Thus, 4-methyl-2-oxopentanoate, 2-oxoisopentanoate, lactate and octanoate were approximated and categorized in the first-degree equation group (Figure 2c). On the other hand, 2-oxoglutarate was categorized in the second-degree equation group (Figure 2d).

We further analyzed the distribution of the compounds that sensitively represented the decline of eGFR in the early stage of CKD. Figure 3 shows the relationship between eGFR and the converted plasma concentration values of the compounds. The concentration of $\mathrm{Cr}$ and cystatin $\mathrm{C}$ approximated by the second-degree equation started to increase from eGFR $30 \mathrm{ml} \mathrm{min}^{-1}$ per $1.73 \mathrm{~m}^{2}$ with an exponential curve. On the other hand, when we chose compounds that significantly correlated with eGFR (rs $>0.7)$ and approximated by the first-degree equation, the concentration of 1 -methyladenosine, $N$-acetylglucosamine, $\gamma$-butyrobetaine, sebacate, cis-aconitate and homovanillate began to increase from eGFR $60 \mathrm{ml} \mathrm{min}^{-1}$ per $1.73 \mathrm{~m}^{2}$ in a linear correlation. 


\section{DISCUSSION}

The accumulation of uremic toxins leads to difficulty in controlling blood pressure and impaired renal function and worsens the prognosis. ${ }^{5,14,30}$ So far, more than 110 organic compounds have been identified as uremic retention solutes. ${ }^{4,5}$ We found 22 cations and 30 anions that accumulated significantly as the eGFR decreases. These compounds included 9 cations and 27 anions that were newly identified in this study. We also found 7 cations and 5 anions, including 2 newly found cations and 5 anions, that decreased significantly as eGFR declined. Thus, knowledge of the retained compounds in CKD could serve as markers for detecting the progression of CKD.

In clinical practice, the plasma $\mathrm{Cr}$ concentration is inversely related to the GFR and used for estimating GFR. However, the serum Cr level does not increase until the GFR has moderately decreased and this is referred to as a 'creatinine-blind GFR area' $\left(40-70 \mathrm{ml} \mathrm{min}^{-1}\right.$ per $\left.1.73 \mathrm{~m}^{2}\right) .{ }^{31}$ Recently, cystatin $\mathrm{C}$ has been a better marker of kidney function. ${ }^{32}$ However, analysis of the data with receiver operating characteristic curves did not show any difference in diagnostic accuracy between cystatin C and Cockcroft and Gault results. ${ }^{32}$ In this study we propose that a number of compounds, all of which have a high correlation coefficients with eGFR (rs >0.7) and whose plasma manner of concentration change is approximated by the first-degree equation increase or decrease in a linear manner from the early stage of $\mathrm{CKD}$, could be better markers for detecting early change of GFR. On the basis of these criteria, 1-methyladenosine, $\mathrm{N}$-acetylglucosamine, $\gamma$-butyrobetaine, sebacate, cis-aconitate and homovanillate could be candidates as markers for detecting the
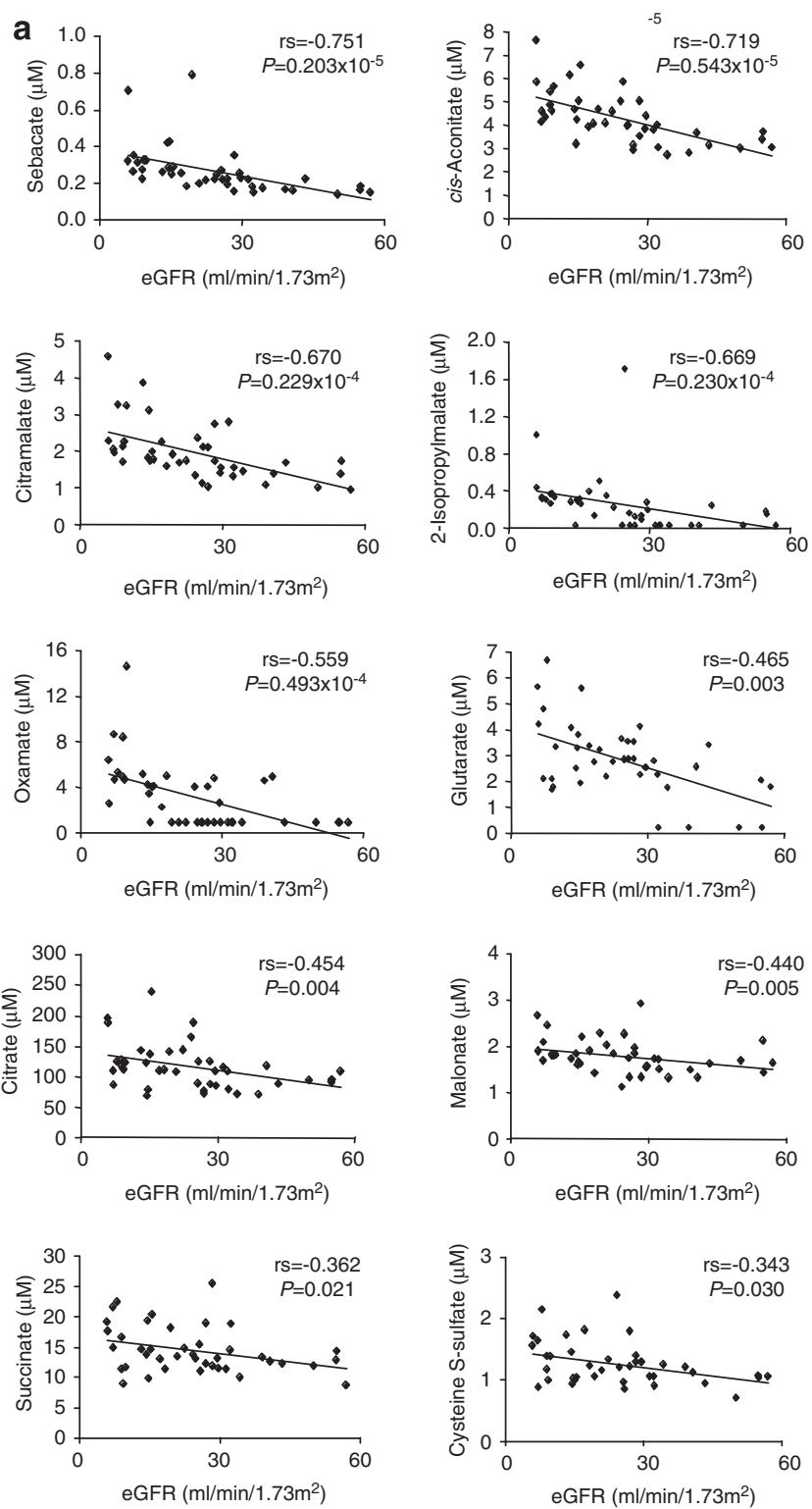
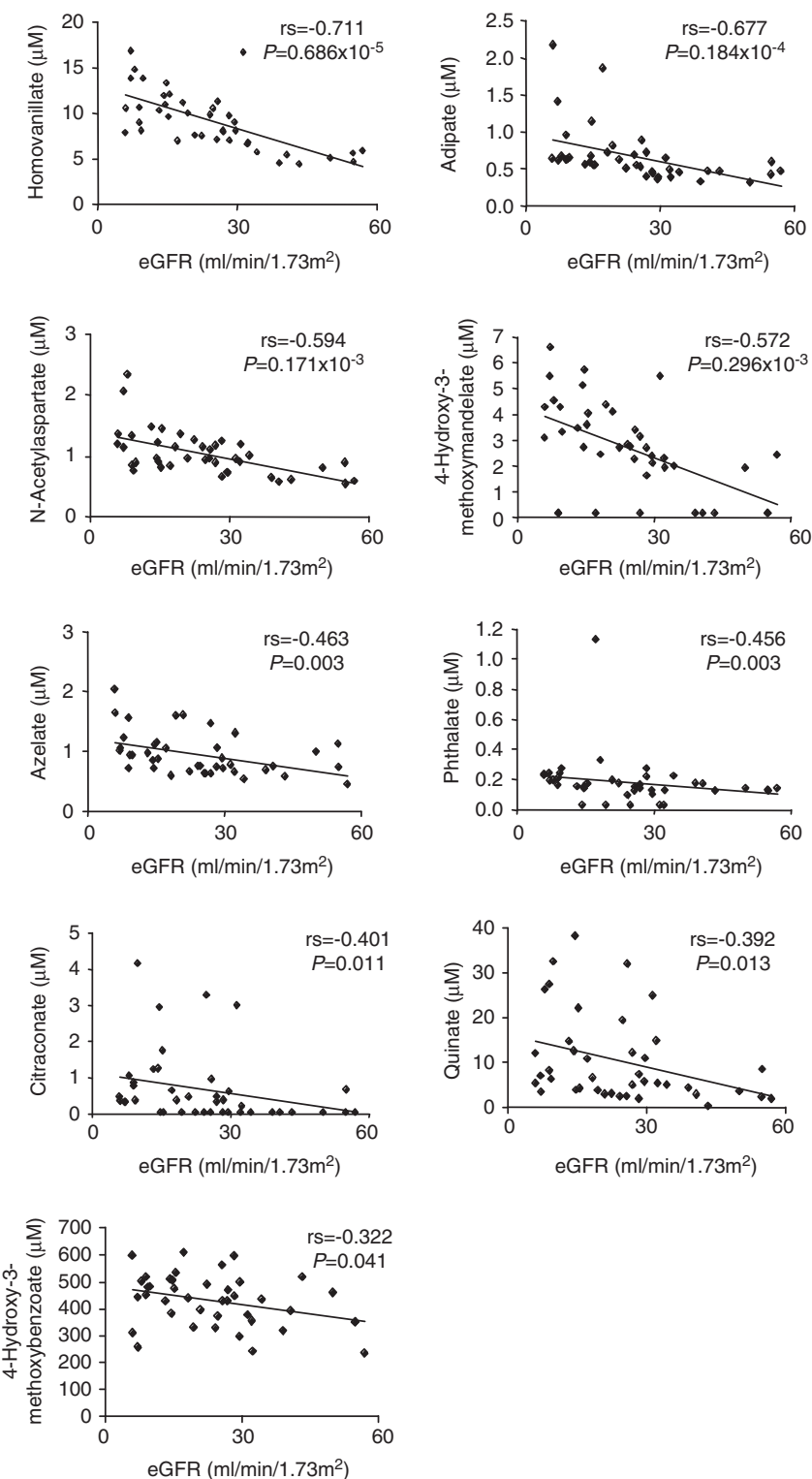

Figure 2 (a, b) The relation between eGFR and plasma concentration of 30 anionic compounds that accumulated significantly as eGFR declined is shown. (a) Compounds that are categorized in the first-degree equation group. (b) Compounds that are categorized in the second-degree equation group. (c, d) The relation between eGFR and plasma concentration of five anionic compounds that accumulated significantly as eGFR declined is shown. (c) Compounds that are categorized in the first-degree equation group. (d) Compounds that are categorized in the second-degree equation group. 

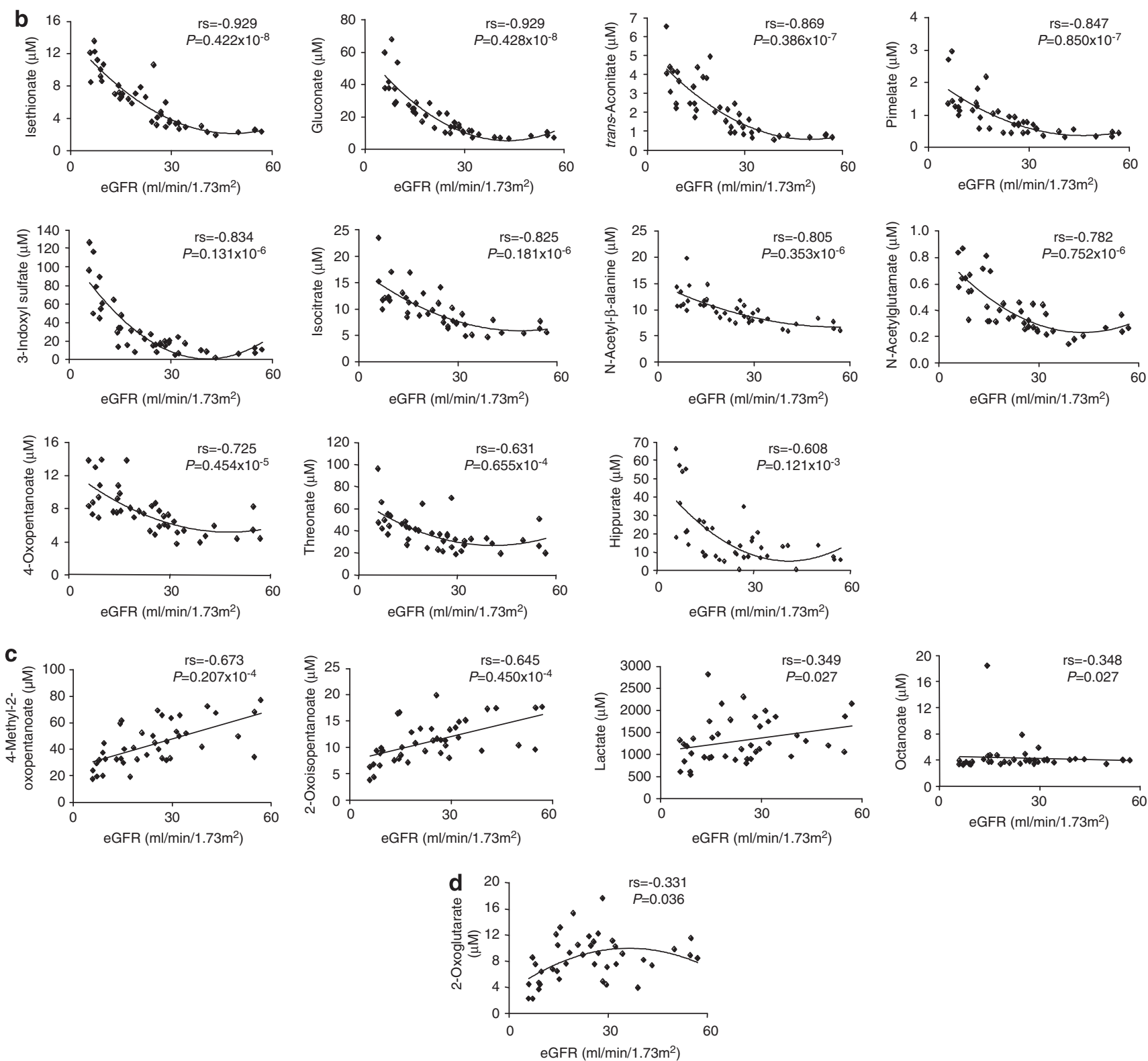

Figure 2 Continued.

early stage of renal damage in CKD patients (Figure 3). In addition, the serum level of 1-methyladenosine has been reported as cancer marker in the patients. ${ }^{33}$

The most important limitation of our analysis is that we did not examine the association of these compounds with age, gender, body weight, cigarette smoking, nutrition status or inflammation. We could not analyze any effects of prescribed drugs or causative diseases because the number of patients was too small to evaluate.

We also found no significant deference between patients with immunoglobulin A nephropathy or diabetic nephropathy and without the diseases. Further experiment should be needed to clarify the effect of drugs. In addition, we used eGFR to estimate the renal function instead of directly measuring GFR by measuring the clearance of exogenous substances such as inulin. We also need to clarify the kinetics, toxicity and metabolic pathway of the compounds. Among the candidate of early marker for CKD patients, 1-methyladenosine is known to accumulate in CKD patients because of lowering of excretion in urine. ${ }^{18}$ However, the exact accumulation mechanisms of the other candidates have not been so far known. Further investigations classifying these compounds according to their pathophysiological importance will be a clue for preserving renal function in CKD patients.

We also detected species differences. As reported, ${ }^{6}$ the concentrations of anionic compounds (indoxyl sulfate and trans-aconitate) were increased in both rat renal failure and CKD patients. On the other hand, increases in 4-acetylbutyrate, hexanoate, 2-hydroxypentanoate and argininosuccinate in rat renal failure were not detected in CKD patients. Among the cationic compounds, the concentrations of asymmetric dimethylarginine, creatinine, guanidinosuccinate, citrulline, 3-methylhistidine, $N, N$-dimethylglycine, allantoin and trimethylamine $N$-oxide were increased in both rat renal failure and in CKD patients. On the other hand, no increases in $\alpha$-aminoadipate and 


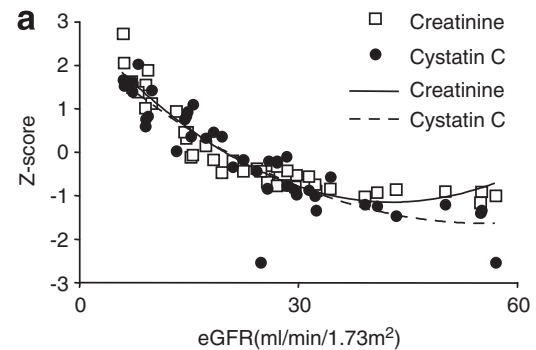

b

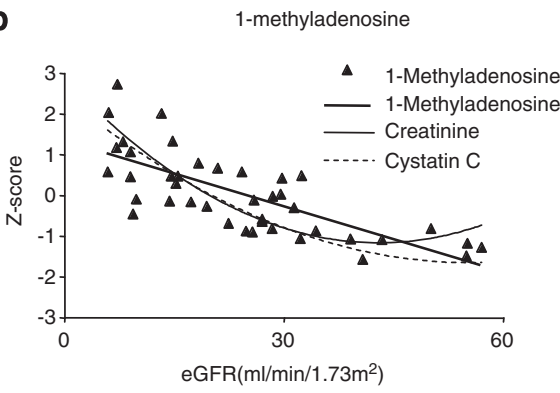

C

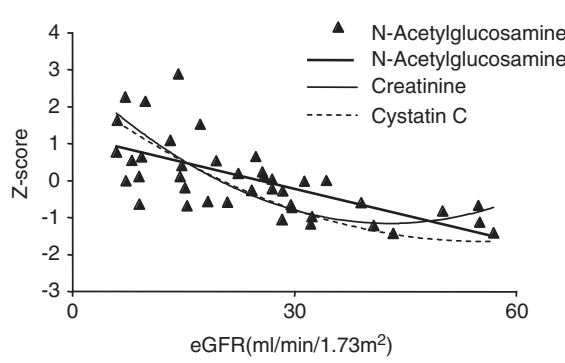

d

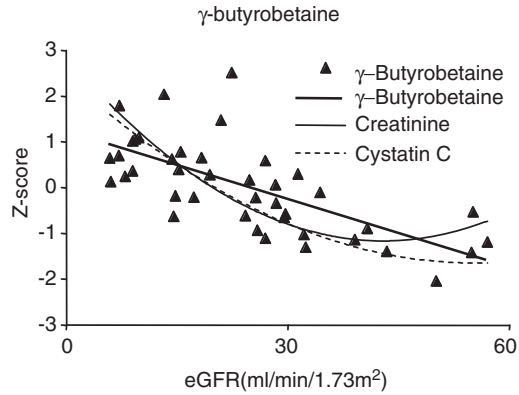

e Sebacate

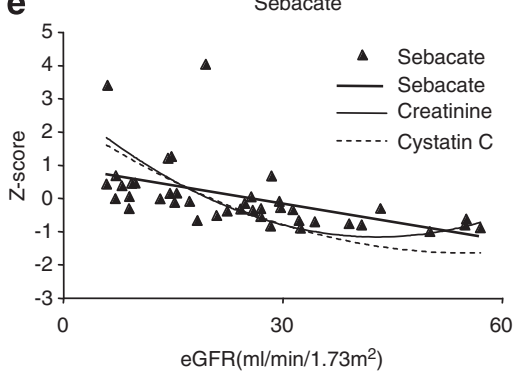

Cis-aconitate

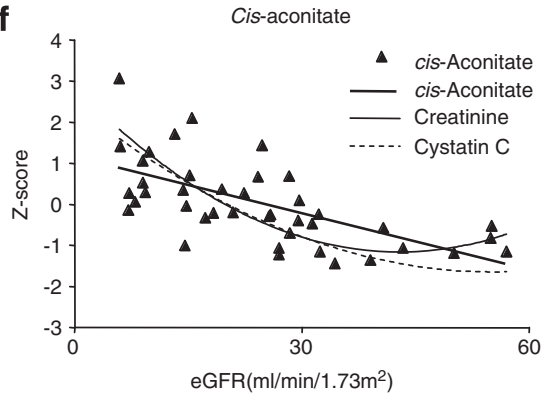

g

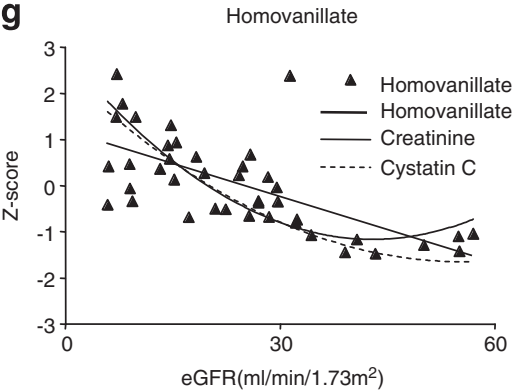

Figure 3 The relationship between eGFR and Z-scores of creatinine (Cr), cystatin C and new uremic marker candidates. (a) Relationship between eGFR and Z-score of creatinine and cystatin C (second-degree group). Approximate curves are shown in solid line (Cr) and dashed line (cystatin C). (b-d) Relationship between eGFR and Z-score of cationic compounds, 1-methyladenosine (b), $N$-acetylglucosamine (c) and $\gamma$-butyrobetaine (d). (e-g) Relationship between eGFR and Z-score of anionic compounds, sebacate (e), cis-aconitate (f) and homovanillate (g).

pipecolate were detected in CKD patients. Furthermore, the concentrations of guanidinoacetate, Trp and Tyr were decreased in both rat renal failure and CKD patients, but carnitine, desethylatrazine and methionine sulfoxide were decreased only in rat renal failure. On the contrary, methionine sulfoxide was increased only in CKD patients. These data suggest careful interpretation of data from rodent experiments into humans.

\section{ACKNOWLEDGEMENTS}

We thank Naohiko Anzai (Kyorin University) for discussion, Izumi Nakamura (Tohoku University) for secretarial assistance and Seth J Karp for the reading of this paper (Harvard Medical School). This work was supported by Miyagi Kidney Foundation, the Ministry of Education, Science and Culture of Japan, the Yokoyama Clinical Pharmacology Foundation and Japan Science and Technology Agency (JST).

1 Cockcroft DW, Gault MH. Prediction of creatinine clearance from serum creatinine. Nephron 1976; 16: 31-41.

2 Levey AS, Bosch JP, Lewis JB, Greene T, Rogers N, Roth D. A more accurate method to estimate glomerular filtration rate from serum creatinine: a new prediction equation. Modification of Diet in Renal Disease Study Group. Ann Intern Med 1999; 130: 461-470.

3 Go AS, Chertow GM, Fan D, McCulloch CE, Hsu CY. Chronic kidney disease and the risks of death, cardiovascular events, and hospitalization. N Engl J Med 2004; 351: 1296-1305.

4 Vanholder R, De Smet R, Glorieux G, Argiles A, Baurmeister U, Brunet P, Clark W, Cohen G, De Deyn PP, Deppisch R, Descamps-Latscha B, Henle T, Jorres A, Lemke HD, Massy ZA, Passlick-Deetjen J, Rodriguez M, Stegmayr B, Stenvinkel P, Tetta C, Wanner C, 
Zidek W, European Uremic Toxin Work Group (EUTox). Review on uremic toxins: classification, concentration, and interindividual variability. Kidney Int 2003; 63: 1934-1943.

5 Vanholder R, Van Laecke S, Glorieux G. What is new in uremic toxicity? Pediatr Nephrol 2008; 23: 1211-1221.

6 Toyohara T, Suzuki T, Morimoto R, Akiyama Y, Souma T, Shiwaku HO, Takeuchi Y, Mishima E, Abe M, Tanemoto T, Masuda S, Kawano H, Maemura K, Nakayama M, Sato H, Mikkaichi T, Yamaguchi H, Fukui S, Fukumoto Y, Shimokawa H, Inui K, Terasaki T, Goto J, Ito S, Hishinuma T, Rubera I, Tauc M, Fujii-Kuriyama Y, Yabuuchi H, Moriyama Y, Soga T, Abe T. SLCO4C1 transporter eliminates uremic toxins and attenuates hypertension and renal inflammation. J Am Soc Nephrol 2009; 20: 2546-2555.

7 Soga T, Heiger DN. Amino acid analysis by capillary electrophoresis electrospray ionization mass spectrometry. Anal Chem 2000; 72: 1236-1241.

8 Soga T, Ueno Y, Naraoka H, Ohashi Y, Tomita M, Nishioka T. Simultaneous determination of anionic intermediates for Bacillus subtilis metabolic pathways by capillary electrophoresis electrospray ionization mass spectrometry. Anal Chem 2002; 74: 2233-2239.

9 Soga T, Ohashi Y, Ueno Y, Naraoka H, Tomita M, Nishioka T. Quantitative metabolome analysis using capillary electrophoresis mass spectrometry. J Proteome Res 2003; 2 : 488-494.

10 Soga T, Igarashi K, Ito C, Mizobuchi K, Zimmermann HP, Tomita M. Metabolomic profiling of anionic metabolites by capillary electrophoresis mass spectrometry. Anal Chem 2009; 81: 6165-6174.

11 Matsuo S, Imai E, Horio M, Yasuda Y, Tomita K, Nitta K, Yamagata K, Tomino Y, Yokoyama $\mathrm{H}$, Hishida A, Collaborators developing the Japanese equation for estimated GFR. Revised equations for estimated GFR from serum creatinine in Japan. Am J Kidney Dis 2009; 53: 982-992.

12 Cox DR. Regression models and life-tables. J Roy Statist Soc Ser 1972; 34: 187-220.

13 Ohkubo T, Imai Y, Tsuji I, Nagai K, Ito S, Satoh H, Hisamichi S. Reference values for 24-hour ambulatory blood pressure monitoring based on a prognostic criterion: the Ohasama study. Hypertension 1998; 32: 255-259.

14 Marescau B, Nagels G, Possemiers I, De Broe ME, Becaus I, Billiouw JM, Lornoy W, De Deyn PP. Guanidino compounds in serum and urine of nondialyzed patients with chronic renal insufficiency. Metabolism 1997; 46: 1024-1031.

15 Tofuku Y, Muramoto H, Kuroda M, Takeda R. Impaired metabolism of guanidinoacetic acid in uremia. Nephron 1985; 41: 174-178.

16 Swendseid ME, Wang M, Vyhmeister I, Chan W, Siassi F, Tam CF, Kopple JD. Amino acid metabolism in the chronically uremic rat. Clin Nephrol 1975; 3: 240-246.

17 Ceballos I, Chauveau P, Guerin V, Bardet J, Parvy P, Kamoun P, Jungers P. Early alterations of plasma free amino acids in chronic renal failure. Clin Chim Acta 1990: 188: 101-108.

18 Niwa T, Takeda N, Yoshizumi H. RNA metabolism in uremic patients: accumulation of modified ribonucleosides in uremic serum. Technical note. Kidney Int 1998; 53: 1801-1806.
19 Shimizu T, Fukagawa M, Kuroda T, Hata S, Iwasaki Y, Nemoto M, Shirai K, Yamauchi S, Margolin SB, Shimizu F, Kurokawa K. Pirfenidone prevents collagen accumulation in the remnant kidney in rats with partial nephrectomy. Kidney Int Supp/ 1997; 63: S239-S243.

20 Bain MA, Faull R, Fornasini G, Milne RW, Evans AM. Accumulation of trimethylamine and trimethylamine- $\mathrm{N}$-oxide in end-stage renal disease patients undergoing haemodialysis. Nephrol Dial Transplant 2006; 21: 1300-1304.

21 Kand'ar R, Zakova P. Allantoin as a marker of oxidative stress in human erythrocytes. Clin Chem Lab Med 2008; 46: 1270-1274.

22 Saito K, Fujigaki S, Heyes MP, Shibata K, Takemura M, Fujii H, Wada H, Noma A, Seishima M. Mechanism of increases in L-kynurenine and quinolinic acid in renal insufficiency. Am J Physiol Renal Physiol 2000; 279: F565-F572.

23 Ludwig GD, Senesky D, Bluemle Jr LW, Elkinton JR. Indoles in ureamia: identification by countercurrent distribution and paper chromatography. Am J Clin Nutr 1968; 21: 436-450.

24 McGregor DO, Dellow WJ, Lever M, George PM, Robson RA, Chambers ST. Dimethylglycine accumulates in uremia and predicts elevated plasma homocysteine concentrations. Kidney Int 2001; 59: 2267-2272.

25 Tanaka A, Takahashi Y, Mizokuchi M, Shimada N, Koide H. Plasma, urinary, and erythrocyte concentrations of guanidino compounds in patients with chronic renal failure. Ren Fail 1999; 21: 499-514.

26 Niwa T, Ise M. Indoxyl sulfate, a circulating uremic toxin, stimulates the progression of glomerular sclerosis. J Lab Clin Med 1994; 124: 96-104.

27 Aoyagi K, Narita M, Mori M, Tatibana M. Regulation of acetylglutamate in the liver: effect of glucocorticoid and renal failure. Contrib Nephrol 1991; 92: 182-190.

28 Farrell PC, Gotch FA, Peters JH, Berridge Jr BJ, Lam M. Binding of hippurate in normal plasma and in uremic plasma pre- and postdialysis. Nephron 1978; 20: 40-46.

29 Biasioli S, Feriani M, Bigi L, Dell'Aquila R, Braganitini L, Chiaramonte S, Fabris A, Brendolan A, Rondo C, Pradella M, Cesaro A, La Greca G. Tricarboxylic acid cycle intermediates in chronic renal failure. Nephrol Dial Transplant 1987; 2: 313-315.

30 Kielstein JT, Zoccali C. Asymmetric dimethylarginine: a novel marker of risk and a potential target for therapy in chronic kidney disease. Curr Opin Nephrol Hypertens 2008; 17: 609-615.

31 Tanaka A, Suemaru K, Araki H. A new approach for evaluating renal function and its practical application. J Pharmacol Sci 2007; 105: 1-5.

32 Hoek FJ, Kemperman FA, Krediet RT. A comparison between cystatin C, plasma creatinine and the Cockcroft and Gault formula for the estimation of glomerular filtration rate. Nephrol Dial Transplant 2003; 18: 2024-2031.

33 Ishiwata S, Itoh K, Yamaguchi T, Sasaki S, Ishida N, Mizugaki M. Serum 1-methyladenosine and pseudouridine as tumor markers in tumor-bearing mice. Yakugaku Zasshi 1995; 115: 523-527. 